www.jmscr.igmpublication.org

Impact Factor 5.244

Index Copernicus Value: 83.27

ISSN (e)-2347-176x ISSN (p) 2455-0450

crossref DOI: http://dx.doi.org/10.18535/jmscr/v4i9.54

Journal Of Medical Science And Clinical Research

\title{
In Hospital Outcome of Subclinical Right Ventricular Involvement in Patients with Acute Inferior Myocardial Infarction
}

\author{
Authors
}

\author{
Ahmad B. Elden, Mahmoud Ashry, Refaat Fathy \\ Critical Care Unit, Internal Medicine Department, Faculty of Medicine, Assiut University \\ Corresponding Author \\ Ahmad B. Elden \\ Assiut University, P.O. Box 71111 Assiut, Egypt \\ Email:ahmadbahie@yahoo.com, Telephone: +20-1009820300, Fax: +20-2333327
}

\begin{abstract}
Objectives: This study aimed to study the in hospital outcome of subclinical right ventricular involvement (ScRVI)in patients with acute inferior wall myocardial infarction(AIMI).

Patients and Methods: We conducted a short prospective studycarried out on 60 patients with acute inferior myocardial infarction, classified into two groups; patients group: AIMIwith ScRVI; inferior or infroposterior acute myocardial infarction (AMI)patients with $\geq 0.1 \mathrm{mV} V_{4} R$ elevation, and control group: AIMI without ScRVI; inferior or infroposterior AMI patients without $\geq 0.1 \mathrm{mV} V_{4} R$ elevation. We followed up both groups during their hospital stay and compared both groups regarding development of complications and mortality.

Results: Patients with ScRVI in AIMIhad more admission hyperglycaemia (268.80 $\pm 81.88 \mathrm{mg} / \mathrm{dl}$ vs. 219.80 $\pm 98.40 \mathrm{mg} / \mathrm{dl}$, $P$ value $=0.003)$, less incidence of complete ST elevation resolution $(S T R)(60.8 \% \mathrm{vs}$.

$80.6 \%$, p value $=0.032)$, more arrhythmic complications $\left(3^{\text {rd }}\right.$ heart block $[H B]: 6.7 \% \quad$ vs. $0.0 \%, p$ value $=0.042$, atrial fibrillation $[A F]: 10.0 \%$ vs. $2.0 \%$, $p$ value $=0.036)$.

Conclusions: We concluded that patients with ScRVI in AIMI had worse in hospital outcome than patients without ScRVI.

Keywords: Subclinical Right Ventricular Involvement, Acute Inferior Myocardial Infarction.
\end{abstract}

\section{Introduction}

Right ventricular myocardial infarction (RVMI) accompanies AIMI in $30 \%$ to $50 \%$ of patients ${ }^{[1]}$. Hemodynamically significant RV infarction clinically present with hypotension, elevated jugular venous pulse (JVP), and occasionally shock, all in the presence of clear lung fields ${ }^{[2]}$. The ST-segment elevation of $\geq 0.1 \mathrm{mV}$ in the right precordial leads $\left(\mathrm{V}_{4} \mathrm{R}\right)$ of the Electrocardiograph (ECG) is a readily available electrocardiographic sign used for diagnosis of RVMI. Hence ECG has always been the first choice of investigation to detect RVMI. Patients with AIMI who have hemodynamically significant RV involvement appear to have a worse prognosis than those who do not have right ventricular involvement ${ }^{[3]}$. However the outcome of ScRVI in AIMI patients is still unidentified. Our aim of this study was to study the outcome of ScRVI in AIMI patients.

\section{Patients and Methods}

We conducted a short prospective observational study with control group, carried out on 60 patients with AMI admitted in internal medicine 
department CCU at Assiut University Hospital, between January 2016 and May 2016. Written consents were obtained from most of the participants; illiterate participants gave their consent by finger prints. We included patients presented by inferior or infroposterior AMI within $12 \mathrm{~h}$ from symptom onset, treated by thrombolytic agents, with at least $50 \%$ resolution of STR and infarct zone comprised at least three hypokineticsegments of LV inferior or infroposterior walls by echocardiography. The diagnosis of inferior or infroposterior AMI was based on prolonged chest pain lasting $\geq 30 \mathrm{~min}$, ST segment elevation $\geq 1 \mathrm{~mm}$ in at least two electrocardiographic (ECG) inferior leads with or without ST segment depression $\geq 1 \mathrm{~mm}$ of $\mathrm{V}_{1}$ to $\mathrm{V}_{3}$, and a more than threefold increase in serum creatine kinase $(\mathrm{CK})$ level $^{[4]}$. RV involvement defined as STE of $\geq 0.1 \mathrm{mV}$ in any number of right precordial leads including $\mathrm{V}_{4} \mathrm{R}$. ScRVI defined as absenceof hypotension and elevated JVP. We then classified reperfused patients into two groups; completely reperfused with resolution of ST elevation by $>70 \%{ }^{[5]}$ and partially reperfused with ST resolution by $>50-70 \%$.We excluded patients with inadequate echocardiographic image quality, age $\geq 85$ years, significant valvular disease, prior Q-wave MI, presence of LBBBand hemodynamic-cally significant RV infarction,clinically presented with hypotension and elevated JVP. All patients received treatment of AMI according to 2007 Focused Update of the ACC/AHA 2004 Guidelines for the Management of Patients with ST-Elevation Myocardial Infarction ${ }^{[6]}$.

All echocardiograms were performed with an HDI 5000 instrument (Philips Medical Systems, Bothell, Washington, USA) equipped with a broad band harmonic transducer. A standard echocardiography was used within the $3 \pm 2$ days of admission based on apical four and two-chamber views; 2D echocardiograms of the LV short axis were recorded at three levels: mitral valve, midpapillary muscle level, and apex. All echocardiograms were analyzed at Assiut University Internal Medicine Echo Laboratory. LV volumes and ejection fraction (EF) was calculated using a modified Simpson's rule. The mean value of three measurements of the technically best cardiac cycles was taken from each examination. To evaluate regional systolic function, the left ventricle was divided according to a 16-segment model as recommended by the American Society of Echocardiography 2002. For each segment, wall motion was scored from 1 (normal) to 4 (dyskinetic) and a global segmental wall motion score index (WMSI) was calculated as the average over 16 segments ${ }^{[7]}$.

RV function was investigated by tricuspid annular plane systolic excursion (TAPSE) and RV fractional area changes (RVFAC) where the mean value of three measurements of the technically best cardiac cycles was taken to measure RV diastolic area and systolic area. RVFAC were calculated as RVFAC $=$ RVDA-RVSA/RVDA \%. Central venous pressure (CVP) was measured by central catheter inserted in the internal JV (routinely inserted in our CCU for hemodynamic monitoring). IVC diameter was measured by convex probe placed in subxephoid position using $\mathrm{M}$ mode measured in inspiration.

\section{Classification of Patients}

We classified our recruit into two groups; patients group: AIMI with ScRVI; inferior or infroposterior AMI patient's with $\geq 0.1 \mathrm{mV} \mathrm{V} \mathrm{V}_{4} \mathrm{R}$ elevation withabsenceof hypotension and elevated JVP, and control group: IWMI without ScRVI; inferior or infroposterior AMI patients without $\geq 0.1 \mathrm{mV} \mathrm{V}{ }_{4} \mathrm{R}$ elevation.

\section{Statistical Analysis}

The statistical analysis wasperformed using SPSS (version 16.0, SPSS Inc., Chicago, IL, USA). The Kolmogorov-Smirnov test was used to test normally. The continuous variables were presented as the means \pm SDand categorical variables were reported as percentages. Student Ttestwas used to compare between means of 2 continuous variables in case of parametric data and Mann-Whitney test for non-parametric data, and chi-square testwas used to compare 
percentages and ratios. Pearson correlation was done to measure correlation among quantitative variables. A p-value $<0.05$ was considered statistically significant.

\section{Results}

\section{Basal Characteristics and Clinical Data}

The study carried out on 60 patients, 30 patients had AIMI with ScRVI and 30 patients had AIMI without ScRVI. The study showed insignificant differences between groups as regard age, gender,BMI and risk factors. There were insignificant differences between groups in admission systolic and diastolic BP. Patientgroup had significantly lower HR than control group (Table 1).

\section{Laboratory data}

There were insignificant differences between groups in peak CK, troponin, LDL, HDL, serum creatinine, BUN. Patients group had significantly higher admission blood glucose (BG) level than control group (Table 1).

\section{ECG Data}

There were insignificant difference between groups in infarction size estimated by number of involved leads and summation of ST elevations in LV leads. There was significantly lower rate of > $70 \%$ resolution in STE in patient group and significantly higher incidences of a trial fibrillation and third degree heart block in patients group (Table 2).

\section{Echocardiographic Data}

There were insignificant differences between groups in EF and WMSI, PASP, incidence of MR, TR, TAPSE and RVFAC.

There were insignificant differences between groups in CVP and IVCD, while patients group had insignificant higher mortality rate (Table 2).

\section{Relations between Sum RST elevation and other hemodynamic}

There were insignificant correlations between summation of right STE and all of; systolic BP, TAPSE, RVFAC, CVP and IVCD(Table 3).

\section{Discussion}

Our study groups were homogenous as regard age, gender, and risk factors, LDL, HDL, serum creatinine, BUN, LV infarction size (ECG, enzymes and echocardiography driven) and LVEF, which reduced confounding factors.

Table (1): Demographic, selected clinical and laboratory data

\begin{tabular}{|c|c|c|c|}
\hline & Patients ${ }^{*}(n=30)$ & $\operatorname{Control}^{+}(n=30)$ & P-value \\
\hline Age(years) $M \pm S D$ & $55.83 \pm 9.44$ & $54.87 \pm 13.35$ & 0.583 \\
\hline MaleNo. $(\%)$ & $23(76.7 \%)$ & $27(90.0 \%)$ & 0.166 \\
\hline Duration of admission(days) $\mathrm{M} \pm \mathrm{SD}$ & $4.73 \pm 1.39$ & $4.53 \pm 1.14$ & 0.095 \\
\hline Hx. DM( $(\%)$ & 63.3 & 40.0 & 0.071 \\
\hline Hx. HTN(\%) & 26.7 & 30.0 & 0.774 \\
\hline $\operatorname{Smoking}(\%)$ & 76.7 & 83.3 & 0.519 \\
\hline $\mathbf{B M I}\left(\mathrm{kg} / \mathrm{m}^{2}\right) \mathbf{M} \pm \mathrm{SD}$ & $30 \pm 2.2$ & $29 \pm 3.1$ & 0.167 \\
\hline Systolic BP(mmhg) M \pm SD & $115.00 \pm 28.13$ & $129.33 \pm 20.16$ & 0.190 \\
\hline Diastolic BP(mmhg $) \mathrm{M} \pm \mathrm{SD}$ & $69.00 \pm 18.45$ & $72.67 \pm 11.17$ & 0.122 \\
\hline Heart rate(beat/ $\min ) \mathrm{M} \pm \mathrm{SD}$ & $71.33 \pm 16.91$ & $82.53 \pm 12.29$ & 0.006 \\
\hline Total $\mathbf{C K}(\mathrm{u} / \mathrm{l}) \mathrm{M} \pm \mathrm{SD}$ & $1129.93 \pm 779.16$ & $1642.60 \pm 1157.86$ & 0.095 \\
\hline $\operatorname{Troponin}(\mathrm{u} / \mathrm{l}) \mathrm{M} \pm \mathrm{SD}$ & $0.93 \pm 0.94$ & $4.25 \pm 8.14$ & 0.264 \\
\hline Random blood sugar $(\mathrm{mg} / \mathrm{dl}) \mathrm{M} \pm \mathrm{SD}$ & $268.80 \pm 81.88$ & $219.80 \pm 98.40$ & 0.003 \\
\hline $\mathbf{L D L}(\mathrm{mg} / \mathrm{dl}) \mathrm{M} \pm \mathrm{SD}$ & $130.93 \pm 37.68$ & $130.12 \pm 43.24$ & 0.667 \\
\hline $\mathbf{H D L}(\mathrm{mg} / \mathrm{dl}) \mathrm{M} \pm \mathrm{SD}$ & $30.90 \pm 8.24$ & $32.87 \pm 8.52$ & 0.372 \\
\hline $\mathbf{B U N}(\mathrm{mmol} / \mathrm{l}) \mathrm{M} \pm \mathrm{SD}$ & $5.99 \pm 3.25$ & $5.58 \pm 3.19$ & 0.673 \\
\hline S. $\mathbf{C R}(\mathrm{mmol} / \mathrm{l}) \mathrm{M} \pm \mathrm{SD}$ & $97.90 \pm 31.93$ & $98.76 \pm 43.25$ & 0.625 \\
\hline
\end{tabular}

* Patients with inferior or infroposterior AMI with $\geq 0.1 \mathrm{mV}$ V4R elevation with absence of hypotension and elevated JVP

${ }^{+}$Patients with inferior or infroposterior AMI without $\geq 0.1 \mathrm{mV}$ V4R elevation

No.: number, h: hour, m; mean, SD; standard deviation, HTN: hypertension, DM: diabetes mellitus, BMI: body mass index, HR: heart rate, BP: blood pressure, S CR: serum creatinine, BUN; blood urea nitrogen, CK; creatine kinase. 
In a systematic overview and meta-analysis of the published literatures, it has been reported that stress hyperglycemia $(\mathrm{SH})$ was associated with an increased risk of mortality in AMI; the association was observed irrespective of diabetic status but was stronger in non-diabetic patients ${ }^{[8]}$. It has been proposed that in AMI patients, decreased levels of blood insulin associated with hyperglycemia may lead to a decrease of glycolytic substrate for cardiac muscle.

Table (2): ECG, echo, IVC, CVP, and mortality data

\begin{tabular}{|c|c|c|c|}
\hline & Patients ${ }^{*}(\mathrm{n}=30)$ & $\operatorname{Control}^{+}(\mathrm{n}=30)$ & P-value \\
\hline \multicolumn{4}{|l|}{ ECG: } \\
\hline Sum LV STE $(\mathrm{cm})(\mathrm{M} \pm \mathrm{SD})$ & $13.5 \pm 4.5$ & $12.5 \pm 5.5$ & 0.063 \\
\hline Number of $L V$ leads $(M \pm S D)$ & $4 \pm 2$ & $4 \pm 3$ & 0.987 \\
\hline Sum RV STE $(\mathrm{cm})(\mathrm{M} \pm \mathrm{SD})$ & $10 \pm 4$ & -- & -- \\
\hline$>70 \%$ STR $(\%)$ & 60.8 & 80.6 & 0.032 \\
\hline \multicolumn{4}{|l|}{ Arrhythmia: } \\
\hline $\mathbf{3}^{\text {rd }} \mathbf{H B}(\%)$ & 6.7 & 0.0 & 0.042 \\
\hline $\mathbf{1}^{\text {st }} \mathbf{H B}(\%)$ & 11.0 & 13.3 & 0.121 \\
\hline Atrial Fib $(\%)$ & 10.0 & 2.0 & 0.036 \\
\hline $\mathbf{V T}(\%)$ & 6.7 & 6.7 & -- \\
\hline \multicolumn{4}{|l|}{ ECHOCARDIOGRAPHY } \\
\hline $\mathbf{E F}(\%)(\mathrm{M} \pm \mathrm{SD})$ & $55.63 \pm 10.22$ & $55.03 \pm 10.41$ & 0.727 \\
\hline WMSI $(M \pm$ SD) & $1.22 \pm 0.15$ & $1.21 \pm 0.16$ & 0.051 \\
\hline $\operatorname{PASP}(\mathrm{M} \pm \mathrm{SD})$ & $38.73 \pm 7.63$ & $37.37 \pm 5.30$ & 0.310 \\
\hline MR No. $(\%)$ & $10(33.3 \%)$ & $8(26.7 \%)$ & 0.573 \\
\hline TRNo.(\%) & $22(73.3 \%)$ & $23(76.7 \%)$ & 0.766 \\
\hline TAPSE $(\mathrm{cm})(\mathrm{M} \pm \mathrm{SD})$ & $1.8 \pm 3.1$ & $1.9 \pm 4.03$ & 0.231 \\
\hline RVFAC $(\%)(\mathrm{M} \pm \mathrm{SD})$ & $50.23 \pm 10.11$ & $52.88 \pm 12.01$ & 0.548 \\
\hline $\mathbf{C V P}(\mathrm{cm})(\mathrm{M} \pm \mathrm{SD})$ & $8.5 \pm 2.5$ & $7.5 \pm 2.5$ & 0.346 \\
\hline IVCD $(\mathrm{cm})(\mathrm{M} \pm \mathrm{SD})$ & $1.8 \pm 0.89$ & $1.7 \pm 0.77$ & 0.652 \\
\hline DeathNo. $(\%)$ & $2(6.7 \%)$ & $0(0.0 \%)$ & 0.472 \\
\hline
\end{tabular}

*Patients with inferior or infroposterior AMI with $\geq 0.1 \mathrm{mV} \mathrm{V4R}$ elevation with absence of hypotension and elevated JVP

${ }^{+}$Patients with inferior or infroposterior AMI without $\geq 0.1 \mathrm{mV}$ V4R elevation

No.: number, m; mean, SD; standard deviation, sum ST; summation of ST segment elevation, LV; left ventricle, RV; right ventricle, STR; ST resolution, HB; heart block, atrial fib; atrial fibrillation, EF; ejection fraction, WMSI; wall motion score index, PASP; pulmonary artery systolic pressure, MR; mitral regurgitation, TR; tricuspid regurgitation, TAPSE; tricuspid annular plane systolic excursion, RVFAC; right ventricle fractional area changes, CVP; central venous pressure, IVCD; inferior vena cava diameter.

Table (3): Correlations between summation of RV STE vs. SBP, TAPSE, RVFAC, CVP and IVCD

\begin{tabular}{lll}
\hline Parameter & r-value & P-value \\
\hline Sum RV STE vs. Systolic BP & 0.237 & 0.868 \\
Sum RV STE vs. TAPSE & 0.199 & 0.675 \\
Sum RV STE vs. RVFAC & 0.439 & 0.661 \\
Sum RV STE vs. CVP & 0.398 & 0.871 \\
Sum RV STE vs. IVCD & 0.076 & 0.876 \\
\hline
\end{tabular}

Sum RV ST; summation of right ventricular ST segment elevation, TAPSE; tricuspid annular plane systolic excursion, RVFAC; right ventricle fractional area changes, CVP; central venous pressure, IVCD; inferior vena cava diameter, SBP; systolic blood pressure.

As a result, the heart has to depend on alternate substrates such as free fatty acids for its metabolism. The accumulation of excessive free fatty acids results in the reduction of myocardial contractility and increases the risk of pump failure and arrhythmias ${ }^{[9]}$. Acute illness, raise levels of counter regulatory hormones resulting in increased hepatic glucose production, impaired peripheral glucose utilization, and relative insulin deficiency ${ }^{[10]}$. In our study, patients with ScRVI had significantly higher admission BG level than patients without ScRVI which can carrya bad 
prognosis.

Monitoring of ST-segment resolution in the surface ECG is the simplest way of determining adequacy of myocardial reperfusion. The most common method of STRclassification is the following: incomplete; ST-segment resolution < $30 \%$, partial; ST-segment resolution $30-70 \%$ and complete; ST-segment resolution $\geq 70 \%{ }^{[11]}$. Angeja et al. showed that angiographic assessment of reperfusion provides information on the mechanical patency of coronary arteries, while the surface electrocardiography reflects the functional status of supplied myocardium ${ }^{[12]}$.

There was significantly lower rate of $>70 \%$ STR in patients with ScRVI. Partial reperfusion with > $50-<70 \%$ resolution in STEis associated with no reflow phenomenon.A series of studies has shown that 'no-reflow' has a strong negative impact upon clinical outcome ${ }^{[13]}$.Patients with 'no-reflow' exhibit a higher prevalence of early post infarct complications, adverse left ventricular remodeling, late repeat hospital stays for heart failure and mortality ${ }^{[14]}$. 'No-reflow' is caused by the variable combination of 4 pathogenic mechanisms. This includes distal atherothrombotic embolization, ischemic injury, reperfusion injury and heightened susceptibility of coronary microcirculation to injury ${ }^{[15]}$.

RVMIs are more often complicated by all types of arrhythmiascompared with 'simple' inferior or anterior wall $\mathrm{LVMIs}^{[16]}$. Barrillon et al ${ }^{[17]}$ were the first to recognize the significantly higherrisk of conduction disorders in patients with RVI. Complete atrioventricular (AV) or sinoatrial blocks occurred in onehalfof cases in which STE in $\mathrm{V}_{4} \mathrm{R}$ were present. Zehender et al ${ }^{[18]}$ demonstrated a higher incidence of sustained ventricular tachycardia and ventricular fibrillation in patients with ECG signsof RVI and significantly higher incidences of complete AV block and severe bradycardias with pacing requirements havebeen reported in cases of RVMI ${ }^{[18]}$. In our study, there were significantly higher incidences in atrial fibrillation and third degree heart block in patients with ScRVI. Patients with ScRVI had insignificantly higher mortality than patients with without. However, the small samplesize and the short duration of follow upcould explain the statistical insignificance.

Higher ST segment elevations in $\mathrm{V}_{4} \mathrm{R}$ have been found to be independent predictive factorsfor more significant RV dysfunction and higher mortality rates ${ }^{[18]}$. In our study, despite there was ECG characters of RVI in patient group, there were insignificant differences between groups regarding; PASP, incidence of MR, TR, TAPSE and RVFAC, CVP and IVCD.Also, our study showed insignificant correlations between summation of right STE vs. systolic BP, TAPSE, RVFAC, CVP and IVCD. This can be explained by that; the summation of right STE were calculated at time of admission and in many cases the RV STE had rapid resolution, which may explain the lake of correlation with hemodynamic and echocardiographic parameters, and a persistent RV STE could explain the worse prognosis in other studies. Furthermore, the small sample size of the groups decreased the study power and increased vulnerability of type II error that may interfere with detection of significant correlation between them.

\section{Conclusion}

We concluded that ScRVIin AIMI had worse prognosis in the form of more admission hyperglycaemia, less incidence in completeSTR, more arrhythmic complications.

\section{Study limitations and recommendations:}

Financial factors interfering with performing a diagnostic coronary angiography in the admission days, so we depended on ST segment resolution by ECG only; this was used to reflect myocardial perfusion rather than epicardial perfusion.A larger sample size is recommended to increase study power and lower type II error. A more prolonged prospective study is recommended for detecting more complications of ScRVI.

\section{Acknowledgements}

The authors wish to acknowledge the staff and workers in the CCU and echocardiography unit of 
internal medicine department at Assiut University Hospitals for their support to this study.

\section{Conflict of interests}

The authors declare that there was no conflict of interests as regard the publication of this paper.

\section{References}

1. Stanley George, Mansi Patel and Ashok Thakkar (2014):Clinical Profile and InHospital Outcome of Patients with Right Ventricular Myocardial Infarction. International Journal of Clinical Medicine; 5, 459-63.

2. Haji, S.A. and Movahed, A. (2000):Right Ventricular Infarction-Diagnosis and Treatment. Clinical Cardiology, 23,473482.

3. Mehta, S.R., Eikelboom, J.W., Natarajan, M.K., Diaz, R., Yi, C., Gibbons, R.J. and Yusuf, S. (2001)Impact of Right Ventricular Involvement on Mortality and Morbidity in Patients with Inferior Myocardial Infarction. Journal of the American College of Cardiology,37,37-43.

4. Elliott M. A, Daniel T. Anbe and Frederick G. Kushner et al. (2004): ACC/AHA Guidelines for the Management of Patients with STElevation Myocardial Infarction. A Report of the American College of Cardiology/American Heart Association Task Force on Practice Guidelines (Committee to Revise the 1999 Guidelines for the Management of Patients With Acute Myocardial Infarction) Developed in Collaboration With the Canadian Cardiovascular Society. American College of Cardiology Web Site (www.cardiosource.org/acc).

5. Schröder K, Wegscheider K and Zeymer $\mathrm{U}$ et al. (2001): Extent of ST-segment deviation in the single ECG lead of maximum deviation present 90 or 180 minutes after start of thrombolytic therapy best predicts outcome in acute myocardial infarction. Zeitschriftfür

Kardiologie; 90:557-67.

6. Elliott M. A, Hand M and Armstrong P. W et al. (2008): 2007 Focused update of the ACC/AHA 2004 guidelines for the management of patients with ST-elevation myocardial infarction: a report of the American College of Cardiology/ American Heart Association task force on practice guidelines. Circulation; 117(2):296-329.

7. Manuel D. Cerqueira, Neil J. Weissman and Vasken Dilsizian et al. (2002): Standardized Myocardial Segmentation and Nomenclature for Tomographic Imaging of the Heart. American Heart Association, Writing Group on Myocardial Segmentation and Registration for Cardiac Imaging.Circulation; 105:539-42

8. Capes SE, Hunt D and Malmberg K et al. (2000): Stress hyperglycemia and increased risk of death after myocardial infarction in patients with and without diabetes: a systematic overview. Lancet; 356: 773-8.

9. Oliver M. F and Opie L. H. (1994): Effects of glucose and fatty acids on myocardial ischemia and arrhythmias. The Lancet; 343(8890):155-58.

10. Umpierrez GE and A.E.K. (2004): ICU care for patients with diabetes. Current Opinion in Endocrinology and Diabetes; 11: 75-81.

11. Bolognese L, Carrabba $\mathrm{N}$ and Parodi $\mathrm{G}$ et al. (2004): Impact of microvascular dysfunction on left ventricular remodelling and long-term clinical outcome after primary coronary angioplasty for acute myocardial infarction. Circulation; 109:1121-6.

12. Angeja BG, Gunda M and Murphy SA et al. (2002): TAIMI myocardial perfusion grade and ST segment resolution: association with infarct size as assessed by single photon emission computed tomography imaging. Circulation; 105:282-5. 
13. Brosh D, Assali AR and MagerA et al. (2007): Effect of no-reflow during primary percutaneous coronary intervention for acute myocardial infarction on six-month mortality. American Journal of Cardiology; 99:442-5.

14. Bolognese L, Carrabba $\mathrm{N}$ and Parodi $\mathrm{G}$ et al. (2004): Impact of microvascular dysfunction on left ventricular remodelling and long-term clinical outcome after primary coronary angioplasty for acute myocardial infarction. Circulation; 109:1121-6.

15. Niccoli G, Burzotta F and Galiuto L et al. (2009): Myocardial no-reflow in humans. Journal of the American College of Cardiology; 54:281-92.

16. Mehta SR, Eikelboom JW and Natarajan MK et al. (2001): Impact of right ventricular involvement on mortality and morbidity in patients with inferior myocardial infarction. Journal of the American College of Cardiology;37:37-43.

17. Barrillon A, Chaignon M, Guize L and Gerbaux A (1975):Premonitory sign of heart block in acute posterior myocardial infarction. British Heart Journal;37:2-8.

18. Zehender M, Kasper W and Kauder E et al. (1993):Right ventricular infarction as an independent predictor of prognosis after acute inferior myocardial infarction. New England Journal of Medicine;328:981-8. 